\title{
Alcances y obstáculos de la accountability mediática en América Latina tras la Tercera Ola de la democracia*
}

\author{
Francisco Javier Tagle Montt ${ }^{*}$ \\ Recibido: 2017-05-27 Enviado a pares: 2017-05-30 \\ Aprobado por pares: 2017-06-18 Aceptado: 2017-07-05 \\ DOI: 10.22395/angr.v16n31a5
}

\begin{abstract}
Resumen
Una de las más clásicas funciones normativas de los medios de comunicación en las democracias es la de ser vigilantes del poder, tanto para servir como defensores de la ciudadanía como para que la opinión pública tome correctas decisiones. El presente trabajo discute desde una posición crítica la accountability mediática en América Latina tras la Tercera Ola de la Democracia hasta el presente, la que si bien ha tenido una importante labor denunciante e investigativa en determinados casos de corrupción política, se postula como limitada por dos razones: la primera, la alta concentración de la propiedad de los sistemas de medios regionales ha provocado un bajo pluralismo informativo que puede poner en duda los verdaderos alcances e intenciones de la vigilancia que realizan los medios al poder y su aporte a las democracias. Por otra parte, y como segunda razón, las reformas de los últimos años llevadas a cabo por algunos gobiernos de liderazgo progresista o populista no han creado un ambiente propicio para esta función, al contrario, han propiciado carencias para la libertad informativa, principio básico para la accountability mediática. A modo de conclusión, se reflexiona sobre la importancia de fomentar el pluralismo informativo y la libertad de expresión como bases para la vigilancia de los medios al poder en la región latinoamericana.
\end{abstract}

Palabras clave: América Latina; democracia; accountability; vigilancia; medios; poder; sistemas; concentración; reformas; populismo

Este artículo se deriva de la tesis doctoral del autor defendida en 2016 en la Universidad de Salamanca (España) titulada "Comunicación en América Latina y el rol de accountability mediática al poder político: casos El Comercio y La República en los Vladivideos y El Mercurio de Santiago y La Tercera en Coimas y MOP-GATE", cuyo director fue el profesor Ángel Badillo.

.. Doctor en Estudios Latinoamericanos por la Universidad de Salamanca, España. Comunicador Social y Periodista por la Universidad de los Andes, Chile. Actualmente es profesor e investigador de la Facultad de Comunicación de la Universidad de los Andes, Chile. fjtagle@uandes.cl 


\title{
Scope and obstacles of media accountability In Latin America after the Third Wave of Democracy
}

\begin{abstract}
One of the most classic normative functions of media in democracies is to be vigilant of power, both to serve as defenders of the citizens and for the public opinion to make correct decisions. This paper discusses from a critical perspective the accountability of the media in Latin America from the Third Wave of Democracy up to date, which has been important in denouncing and investigating certain cases of political corruption, but has been limited for two reasons: the first is the high concentration of ownership of regional media systems, have led to a very low level of informative pluralism which calls into question the true scope and intentions of the surveillance that the media can carry out of power and its contribution to democracy. On the other hand, the second reason is the reforms carried out in recent years by the leadership of some progressive or populist governments that have not created a proper environment for this function, and on the contrary, have provided shortcomings for the freedom of information, a basic principle for media accountability. As a conclusion, this paper reflects on the importance of promoting informative pluralism and freedom of expression as the basis of media surveillance of power in the Latin American region.
\end{abstract}

Keywords: Latin America; democracy; accountability; monitoring; media; power; systems; concentration; reforms; populism.

\section{Alcances e obstáculos do accountability midiático na América Latina após a Terceira Onda da Democratização}

\begin{abstract}
Resumo
Uma das mais clássicas funções normativas dos meios de comunicação nas democracias éa de ser vigilantes do poder, tanto para servir como defensores da cidadania quanto para que a opinião pública tome decisões corretas. Este trabalho discute, sob uma posição crítica, o accountability midiático na América Latina após a Terceira Onda da Democratização até o presente, a que, embora tenha tido um importante trabalho denunciante e investigativo em determinados casos de corrupção política, apresenta-se como limitada por duas razões: a alta concentração da propriedade dos sistemas de meios regionais que provoca um baixo pluralismo informativo que pode colocar em dúvida os verdadeiros alcances e intenções da vigilância realizados pelos meios ao poder e sua contribuição para a democracia; por outro lado, e como segunda razão, as reformas dos últimos anos realizadas por alguns governos de liderança progressista ou populista que não criaram um ambiente propício para essa função; ao contrário, promoveram carências para a liberdade informativa, princípio básico para o accountability midiático. Como conclusão, reflexiona-se sobre a importância de fomentar o pluralismo informativo e a liberdade de expressão como bases para a vigilância dos meios ao poder na região latino-americana.
\end{abstract}

Palavras-chave: accountability, América Latina, concentração, democracia, meios, poder, populismo, reformas, sistemas, vigilância. 


\section{Introducción}

De acuerdo con el informe de Transparency International (2016), dos grandes fenómenos ocurrieron en Latinoamérica en el último tiempo: el primero, es que diferentes redes de corrupción política quedaron descubiertas, y segundo, importantes protestas ciudadanas se levantaron exigiendo transparencia a sus autoridades. Si bien cada caso responde a un determinado contexto político del país en que aconteció, es posible establecer un factor común que está dado por el rol que jugaron en ellos los medios de comunicación.

En esta región, y tras las transiciones a la democracia enmarcadas en la Tercera Ola de Huntington (1991), los medios de comunicación tuvieron en algunas ocasiones un rol importante en hacer rendir cuentas al poder político, tanto siendo ellos los protagonistas de las acusaciones como dando cobertura y encuadre a las ilegalidades (Salwen y Garrison, 2014; Waisbord, 2012, 2000, 1996; Huges, 2006; Peruzzotti, 2006; Perruzzotti y Smulivitz, 2006, 2002). Estos casos de denuncias mediáticas pueden explicar por qué la ciudadanía latinoamericana tendió en los últimos lustros a confiar al menos, más en los medios -los denunciantes- que en alguno de los tres poderes del Estado -generalmente, los denunciados- (gráfica I).

Aunque es válido insertar tanto en las teorías de las democracias como en el ámbito de los estudios de la comunicación política a los medios como vigilantes del poder, resulta ingenuo ponderarlos como paladines altruistas y neutrales, y no considerar que son actores que representan determinados intereses políticos y económicos, y cuya importancia social es también apetecida por los gobiernos.

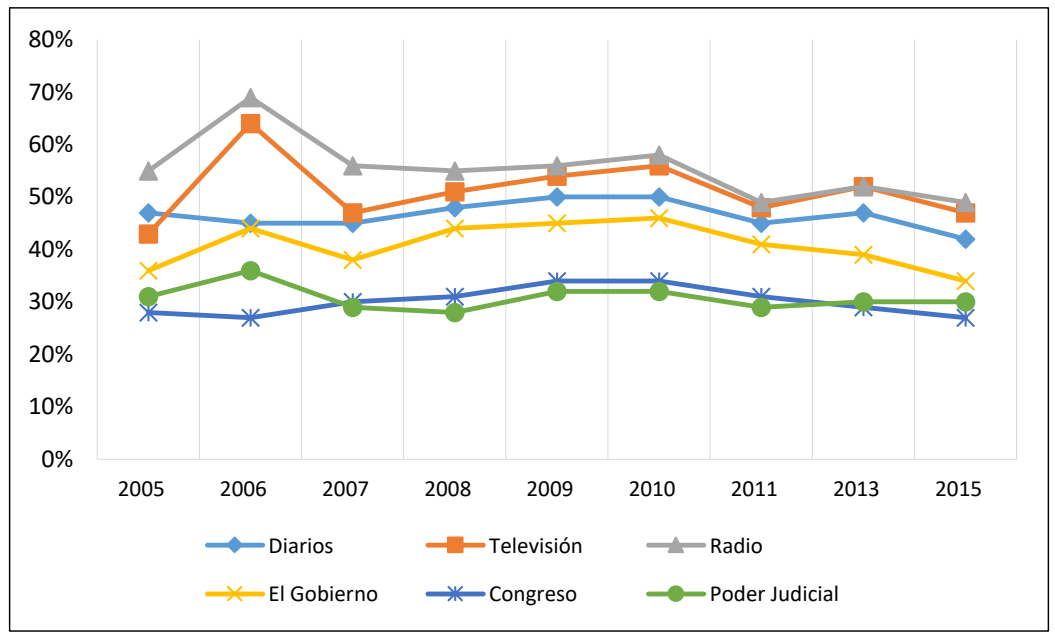

Gráfica I. Porcentajes de confianza a nivel latinoamericano en los diarios, la televisión, la radio, el gobierno, el congreso y el poder judicial entre los años 2005 a $2015^{1}$

Fuente: Latinobarómetro

Para realizar esta comparación entre las distintas instituciones políticas y los medios se sumaron la respuesta de confianza "mucha" y "algo" como una sola, para así mostrar los porcentajes de aceptación de manera más amplía. 
Así, la evaluación de los medios resulta compleja en América Latina respecto a la función de vigilancia. Por ejemplo, mientras en Chile, en 2015, fue una investigación mediática la que dio a conocer el tráfico de influencias del hijo de la presienta Bachelet, unos pocos meses después en Brasil, autores como Van Dijk (2016) y Goldstein (2015) cuestionaron el rol de los medios como contrapoder, debido al papel que tuvieron en la polarización de la crisis política que llevó a la destitución de Rousseff.

En primer lugar, este trabajo discute sobre las causas de algunos casos en que los medios sí ejercieron una función de vigilancia del poder político en América Latina. En segundo término, se abordan los obstáculos que existen en la región en cuanto a sus sistemas de medios para la realización de esta accountability mediática, lo que cuestiona sus verdaderos alcances y aportes: 1) Pluralismo denunciante y 2) Populismos y reformas.

La literatura que ha abordado la problemática de los sistemas de medios latinoamericanos lo ha hecho, generalmente, desde la perspectiva de que la concentración de la propiedad impide un mayor pluralismo de la información y margina a las voces disidentes, obviando o dejando, como un asunto menor, que estas falencias también influyen para que los medios realicen una correcta función de vigilancia, cuestión que se ha asociado siempre más con la tradición anglosajona que con la realidad de esta región.

Como marco para el debate propuesto, resulta propicio comenzar con la discusión teórica acerca de la función normativa de la accountability mediática y su relación con los sistemas de medios.

\section{Marco teórico}

La centralidad que juegan los medios de comunicación en una sociedad se debe, entre otras razones, a su relación con el poder. Desde una interpretación foucaultiana, el poder no se posee, sino que se ejerce y está en una relación de constante circulación. Así, en la sociedad existen diferentes "mecanismos secretos" por lo cuales se transmiten los saberes que perpetúan las relaciones de poder. Entre estos mecanismos estarían los medios de comunicación, puesto que ellos ayudan a controlar el discurso, y, por lo tanto, el saber y su apariencia (Foucault, 1979; 1992). De esta manera, entre los diferentes discursos que los medios realizan en la sociedad, puede estar el de ser vigilantes, que tal como señala McQuail (2013), es la función más deseable y benigna que pueden realizar en las democracias. Normativamente se espera que los medios se adjudiquen la representación de la ciudadanía y su protección respecto a poderes que, supuestamente, son mucho mayores y que atentan en contra de esta.

Fue en las tradiciones norteamericanas liberales en donde esta visión de los medios como contrapoder tuvo su mayor desarrollo (Curran, 2002). Mientras que en el siglo XIX, Tocqueville (1835-1840) estableció a los medios como la mejor defensa en contra de los poderosos, a principios del XX, Lippmann (1922) los definió como "guardianes" de las instituciones, y a mediados de este, Lasswell (1948), como reveladores de amenaza y oportunidades. 
Si bien ha recidido nuevas formulaciones, la función normativa de los medios como denunciantes del poder político continúa en la actualidad como una de las principales herramientas de las democracias para que los ciudadanos participen de manera informada en ellas (Christians, Glasser, McQuail, Nordenstreng y White, 2009; Allen y Blank Hindman 2014; Hampton, 2010). Es lo que se ha teorizado desde la politología como accountability social, haciendo referencia a que la rendición de cuentas en una democracia puede ser llevada más allá de las instituciones del Estado, estableciéndola también, como una relación vertical con el poder que proviene desde la sociedad, y en este caso, respecto al actuar vigilante de los medios (Fox, 2015; Bertot, Jaeger y Grimes, 2012; Peruzzotti y Smulovitz, 2006, 2002; O’Donnell, 2004, 1996).

Si la racionalidad y la acción de la política llevan a la construcción de instituciones que hacen ser funcionales a las ideas, los valores y las aspiraciones de la sociedad (Auat, 2003), desde la función normativa de la vigilancia mediática se podría argumentar que los medios se preocuparían de asegurar que esas instituciones, desde su ideal burocrático (Weber, 1922), cumplan la misión para la que fueron creadas.

De acuerdo con Thompson (2001), siempre el wachtdog dependerá de la iniciativa individual de medios y periodistas que, más allá del sensacionalismo y réditos comerciales que producen los escándalos mediáticos, creen que con su labor pueden reformar a la sociedad. Así, teniendo presente que siempre se tratará de actos mediáticos individuales, no se puede obviar que existen factores que contribuyen a un ambiente más propicio para la vigilancia de estos en las sociedades.

Desde la perspectiva de la relación entre el sistema de medios y la accountability mediática -cuestión que es la que discute este trabajo- y teniendo presente que los medios son mecanismos de poder (Foucault, 1979; 1992), el pluralismo externo (Hallin y Mancini, 2004), entendido como aquellos sistemas en donde existen una serie de medios que representan diferentes puntos de vistas, resultaría ser más propenso para la vigilancia del poder (Prat y Stromberg, 2013). Si bien Christians et al. (2009) postulan que el ideal normativo de las denuncias mediáticas es que deben ser equilibradas, sin distinción política y abiertas a la defensa de los acusados, pareciera que esto es más probable de lograr en sistemas compuestos por un abanico amplio de medios que representan distintas posiciones ideológicas, más que en los sistemas de pluralismo interno (Hallin y Mancini, 2004), dado que en ellos se pone toda la confianza en que cada medio es neutral y capaz de tener veracidad y equilibrio al momento de informar, lo que la realidad se ha encargado de poner generalmente en duda.

Como señalan Gentzkow y Shapiro (2008, p. p.133) "los mercados de las noticias promueven la verdad". En este sentido, los intentos de manipulación de la información que puedan existir en los medios para llevar a cabo determinadas agendas de poder, tanto políticas como económicas, se disminuye cuando existen variados puntos de vista en el sistema. 
Por otra parte, Vaidya y Gupta (2016) explican que en su relación con la autoridad política, la independencia de los medios como vigilantes puede verse comprometida, no solo a través de legislaciones que permitan el manejo directo de medios de propiedad estatal y el control de los privados, sino también mediante sobornos. Besley y Prat (2006) sostienen que el pluralismo informativo provee una protección efectiva en contra de la posibilidad de captura del poder político de los medios. Esto porque un mayor pluralismo mediático en una democracia hace más difícil la tarea de las autoridades corruptas de impedir, ya sea mediante favores o sobornos, la información que les puede ser perjudicial, dado que el medio que no informa sobre un determinado tema da una ventaja comercial a otro que sí lo hace.

\section{Discusión: Los medios de comunicación como contrapoder en América Latina, sus alcances y limitaciones}

Con las transiciones a la democracia que vivieron la mayoría de los países en América Latina a partir de la década de los ochenta, comenzó un periodo democrático único en su historia: nunca antes, tantos de sus países y por tanto tiempo han estado bajo este régimen político (Mainwaring y Pérez-Liñán, 2014). Sin embargo, una de las principales deficiencias que se han diagnosticado a estas democracias ha sido la falta de adecuados mecanismos para que el poder rinda cuentas (Pasquino, 2015; Alcántara, 2006, 2012; Del Tronco, 2013; Mainwaring, 2003; O’Donnell, 1994, 2004).

La fiscalización y vigilancia que pueden hacer los distintos poderes del Estado presentan dudas en América Latina, debido a los altos niveles de corrupción que se han mantenido por décadas. No solo varios de sus países se sitúan entre los más corruptos del mundo, sino que las instituciones encargadas de vigilar y fiscalizar poseen un fuerte descrédito ciudadano, por lo que resulta contradictorio que sean estas las llamadas a resguardar la probidad (Latinobarómetro, 2015; Transparency International, 2016).

Por otra parte, son numerosos los casos en la región de políticos que fueron protagonistas de actos de corrupción que después fueron nuevamente electos o gozan de una alta popularidad. En este sentido, el retorno del sufragio como mecanismo de rendición de cuentas ha mostrado falencias, especialmente, por la naturaleza limitada del voto, dado que concede solamente una posibilidad para sacar o recompensar numerosas decisiones gubernamentales, y en promedio, los votantes presentan un déficit de información (Peruzzotti y Smulovitz, 2006).

Este escenario ha sido el que ha permitido que los medios de comunicación en Amér rica Latina hayan cobrado mayor importancia como agentes de vigilancia del poder. Por ejemplo, entre los casos más emblemáticos ocurridos en algunos países latinoamericanos durante la década de los noventa y comienzos de los 2000 se encuentran:

- Argentina: Las investigaciones de Clarín sobre la venta ilegal de armas a Croacia y a

Perú terminó en una acusación judicial por asociación ilícita en contra del presidente Menem. 
- Brasil: Las denuncias de Folha, el semanario Isto Éy la revista Veja fueron determinantes para el proceso de impeachment en 1992 en contra del presidente Collor de Mello.

- Colombia: Durante los noventa, los periódicos El Tiempo, El Espectador, Vanguardia Liberal y El Colombiano se unieron en una cruzada para denunciar los lazos del mundo político con el narcotráfico.

- Costa Rica: En 2004, los reportajes del diario La Nación sobre sobornos recibidos por el expresidente Miguel Ángel Rodríguez lo llevaron a renunciar a su cargo de Secretario General de la Organización de Estados Americanos.

- Chile: En 2002 y 2003, el bullado caso de irregularidades en las licitaciones del Ministerio de Obras Públicas se debió a un reportaje de la revista Qué Pasa.

- México: Durante el sexenio de Salinas de Gortari, diarios como Reforma, El Financiero, El Economista y Proceso tuvieron un importante rol denunciando una serie de ilícitos ocurridos en su período.

- Nicaragua: En 2001, el diario La Prensa llevó el caso de los beneficios al margen de la ley que obtuvieron familiares del presidente Arnoldo Alemán.

- Perú: Diarios como El Comercio y La República tuvieron un importante rol denunciando los actos de corrupción del gobierno de Fujimori y su asesor Montesinos.

- Venezuela: En 1992, las denuncias por malversación de fondos realizadas por El Universal en contra del presidente Carlos Andrés Pérez terminaron por destituirlo de su cargo.

Aunque resulta exagerado proponer una tradición de periodismo de vigilancia en esta región (Santoro, 2008), estos importantes y mediáticos casos en los años que siguieron al retorno de la democracia y que impactaron a la opinión pública, ayudaron a que los tribunales de justicia actuaran. Cárdenas y González (2006) denominaron a estos acontecimientos como los Watergates Latinoamericanos en alusión al famoso caso de investigación periodística ocurrido en Estados Unidos, que terminó en la dimisión del presidente Richard Nixon en 1974.

Fueron determinadas condiciones históricas, políticas y económicas las que propiciación este afán de denuncia mediática. El contexto que trajo consigo la Tercera Ola (Huntington, 1991) contribuyó a la existencia de unos medios de comunicación más inquisidores del poder político. El término de las dictaduras militares permitió el fin de la censura y la persecución a periodistas, y así, un ambiente más propicio para la vigilancia mediática. Esto teniendo en perspectiva, que esta región aún carece en la actualidad de una adecuada libertad de prensa (Freedom House, 2016a).

Mientras que los medios alternativos durante los regímenes militares, especialmente del Cono Sur latinoamericano, tuvieron una labor valiente en las denuncias de violaciones a los Derechos Humanos, con el retorno de la democracia, este afán vigilante se trasladó 
a los grandes y comerciales medios de comunicación que lo llevaron a otros planos como la corrupción. Muchos medios vieron en los escándalos mediáticos tanto una posibilidad de aumentar sus audiencias y réditos comerciales -dado que los escándalos venden-, como en una posibilidad de transformarse en verdaderos contrapoderes de los gobiernos (Waisbord, 2000, 2012).

También colaboró para este ambiente vigilante la desregulación de los sistemas de medios en la región. A diferencia de Estado Unidos y Europa, los medios de América Latina hasta los noventa fueron, generalmente, muy cercanos a los gobiernos, quienes en muchos casos tanto ayudaban a financiarlos como los favorecían con las leyes (Fox y Waisbord, 2002). Desde posiciones más complacientes -en los apartados siguientes se discutirán las críticas-, la desregulación de los mercados de la información en América Latina, permitió la consolidación comercial de un grupo importante de medios de comunicación, los que se hicieron más poderosos e independientes económicamente frente a los gobiernos. Como señala Curran (2002), los medios al alejarse del Estado aseguran su independencia y su capacidad de "mordedura" del poder, y esto al parecer, fue el caso de América Latina.

La contracara de este panorama propuesto presenta una serie de obstáculos que contravienen los alcances reales de la vigilancia de los medios en América Latina. Más allá de discutir las intenciones de los medios como contrapoder de los corruptos, los siguientes apartados debaten sobre los sistemas de medios regionales y sus reformas de los últimos años, puesto que allí estarían la claves para promover un correcto ambiente para la accountbility mediática.

\section{Pluralismo denunciante}

Desde el retorno de la democracia a América Latina, las políticas para los sistemas de medios fueron en líneas generales no tener una, lo que ha ido provocando hasta la actualidad, y casi sin excepción de ningún país, una altísima concentración de la propiedad, creando verdaderos imperios de la comunicación, en las que se ha comprendido a los medios como un negocio y no como un bien público, todo acompañado por una trama compleja de intereses empresariales (Becerra, 2014; Becerra y Mastrini, 2006; Boas, 2012; Trejo, 2010; Fox y Waisbord, 2002; Guerrero, 2014).

Aunque la teoría tratada en los apartados anteriores postula que la competencia asegura un ambiente propicio para una correcta rendición de cuentas mediática (Prat y Stromberg, 2013), la liberación de los mercados en la región no fomentó este principio.

Si nos ceñimos a los espacios de información periodística de los medios de comunicación, que es en donde ocurre, generalmente, la accountability mediática, por ejemplo, tenemos que en México, los noticieros de Televisa y Televisión Azteca en horario prime poseen juntos más del 70\% de la audiencia de la televisión abierta (Media Research Center, 2016). Mientras que en Brasil, los conglomerados que están detrás de los dos periódicos políticos más importantes, O Globo y Folha de Sao Paulo, son propiedad de siete familias que concentran el 90\% de los medios de todo el país (Reporteros sin Fronteras, 2016b). 
Asimismo, a pesar de las amplias posibilidades de pluralismo que ofrece Internet, el 31\% de las noticias online son vistas por los brasileros en un solo medio que pertenece a Folhapar (Noam y Mutter, 2016). Por su parte, en Argentina, el grupo Clarín tiene una concentración vertical que lo lleva a tener no sólo el diario político con mayor circulación del país (Instituto Verificador de Circulaciones, 2016), sino que, además, su canal de televisión abierta posee el noticiero del horario prime con mayor rating (Televisión.com.ar, 2016). En Chile, de los cuatro periódicos de circulación nacional, dos pertenecen al grupo El Mercurio SAP, que juntos suman un índice de lectoría del 58\%, mientras que los otros dos diarios pertenecen al conglomerado que le compite, Copesa, que posee el resto (Valida, 2016). En lo que se refiera a Perú, el grupo El Comercio controla el 50\% de la venta de diarios del país y el 80\% de los periódicos que se venden en Lima (Távara, 2016). En tanto, en Colombia, el noticiario de televisión Caracol Noticias posee el 72\% de la audiencia en cuanto a contenido de información y opinión (EGM, 2016).

Esta falta de competencia de los sistemas de medios latinoamericanos respecto a los espacios de información periodística cuestiona un ambiente propicio para la accountability mediatica. De acuerdo con Kitzberger (2016), los grandes conglomerados mediaticos de la región se han ido transformado en actores protagonistas del escenario político, pero no en cuanto a su labor de vigilancia, sino en ser ellos una suerte de pieza clave para la gobernabilidad.

Los grandes conglomerados de medios de comunicación constituyen actores estratégicos para gobernar. Ya que concentran el control sobre los recursos claves (las ideas, información, las agendas públicas, legitimidad y reputación) y tienen la capacidad de actuar en bloque para perseguir sus intereses, tienen el potencial de influir en la formulación de las decisiones políticas y, finalmente, la capacidad de un gobierno para mantenerse en el poder (Kitzberger, 2016, p. 450).

La alta concentración resulta tan dañina para la vigilancia mediática, que la poca competencia que pueda existir en estos sistemas concentrados se diluye. Becerra (2014, p. 64) señala que "la concentración de la propiedad en pocos grupos tiende a la unificación de la línea editorial y a la reducción de la diversidad". Esto se produce debido a que las posiciones económicas de poder de los grandes medios en cuanto, por ejemplo, a vender avisaje de pago, lleva a los pequeños a plegarse más que a la diferenciación para comercializar también publicidad a los mismos anunciantes. En definitiva, como explican Loreti y Lozano (2014), para el caso latinoamericano, este bajo pluralismo mediático tiene como consecuencia la homogeneización de contenidos, en pos de consolidar alianzas comerciales y políticas.

Por otra parte, son numerosos los casos en la región de magnates de las comunicaciones que han ingresado al mundo político, lo que cuestiona también la pretendida independencia de los medios y su vigilancia (Becerra, 2014). Por ejemplo, en Brasil se calcula que unos cuarenta parlamentarios tienen influencia directa, al menos, en un canal de televisión o radioemisora (Reporteros sin Fronteras, 2016b). 
Como se ha señalado, la vigilancia mediática se basa en el postulado liberal de que los medios deben estar lo más alejados del poder político (Curran, 2002), por lo que la dependencia de la publicidad estatal los puede hacer presa fácil para la manipulación. De acuerdo con UNESCO (2104, p.15), "en una gran cantidad de países de la región los medios tradicionales han dependido fuertemente de la publicidad estatal para sobrevivir (...) lo que los hace especialmente vulnerables a la influencia de funcionarios y autoridades". Por ejemplo, en Chile la ley que regula la publicidad estatal es bastante ambigua; así en 2013 se repartieron más de 50 millones dólares entre los principales medios de comunicación sin ninguna especificación del criterio de selección (Fernández, 2014), mientras que en México, a pesar de la reforma electoral de 2007-2008 que impedía la compraventa de espacios en medios para la promoción electoral, la realidad ha sido que persiste un propaganda política encubierta que moviliza varios millones de dólares (Galarza, 2014).

\section{Populismo y reformas}

Desde la década del 2000, algunos gobiernos latinoamericanos de izquierda categorizados como de liderazgo populista (Freidenberg, 2007), y también otros de líneas más socialdemócrata (Weyland, 2010) han intentado realizar reformas encaminadas a dar mayor peso a los medios estatales junto con regular a los medios privados y facilitar la creación de medios comunitarios (Gómez y Ramos-Martín, 2014; Badillo, Mastrini, y Marenghi, 2015; Becerra, 2011). Todo esto, bajo una inspiración que hace correspondencia con las premisas de los estudios de la comunicación latinoamericana de los años sesenta y setenta, las que se sustentaban en criticar a la dominación cultural tanto interna, la elite empresarial, como externa, Estados Unidos (Beltrán, 2008).

Así, estos gobiernos de izquierdas en sus distintas versiones pusieron nuevamente en el centro de la discusión a los medios y su rol democrático. Entre las reformas más importantes están la Ley de Servicios de Comunicación Audiovisual en Uruguay (2014), la Ley de Servicios de Comunicación Audiovisual (2009) en Argentina, la Ley General de Telecomunicaciones, Tecnología de la Información y Comunicación (2011) en Bolivia, la Ley Orgánica de la Comunicación (2013) en Ecuador y la Ley de Responsabilidad Social en Radio y Televisión (2004), también conocida como "Ley Resorte", en Venezuela, a la que se suma, en la misma línea, la discusión comenzada en 2015 en el Congreso brasileño. De acuerdo con Santander (2014), todas estas reformas han sido enfocadas en comprender a la libertad de expresión e información no solo como la ausencia de censura, que es la manera tradicional de concebir este derecho, sino mediante la actuación positiva del Estado frente a la concentración.

No obstante, estas medidas han presentado la problemática de que sus adherentes las consideran como necesarias y urgentes para pluralizar a los medios, mientras que sus detractores las acusan de tener como fin aumentar el poder comunicacional de estos gobiernos (Badillo et al., 2015). Esto último fundamentado en que las administraciones de liderazgo populista se han caracterizado por conflictos con la libertad de prensa, lo que ha ido desde medidas arbitrarias en contra de medios opositores (Amado, 2014), apoyo 
con publicidad estatal a medios afines e incluso leyes dentro de estas mismas reformas que coartan la expresión (Cañizales y Matos-Smith, 2015; Freedom House, 2016a; Reporteros sin Fronteras, 2016a).

En el siguiente cuadro se puede observar cómo se ha ido mermando, desde el 2000 hasta el presente, la libertad de prensa en aquellos países latinoamericanos que emprendieron reformas a sus sistemas de medios.

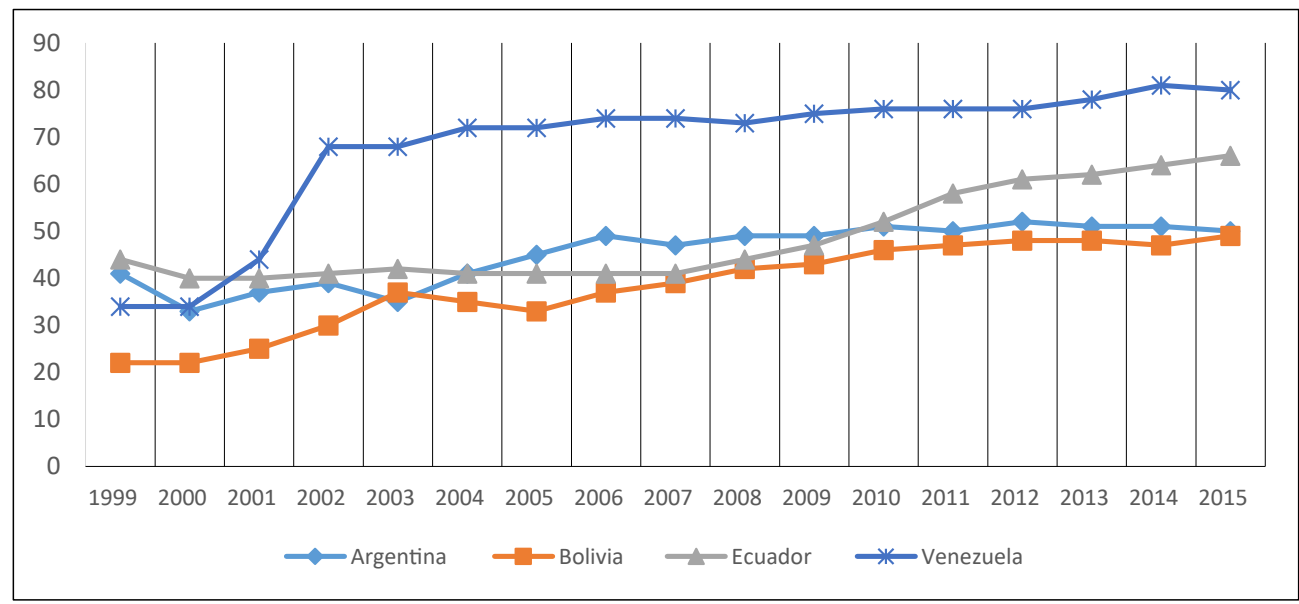

Gráfica II. Puntuación y estatus de acuerdo a Freedom House de la libertad de prensa entre 1999 a 2015 en Argentina, Bolivia, Ecuador y Venezuela (Leyenda: 0-30: Parcialmente Libre; 31-60; No libre: 61-100)

Fuente: Freedom House, 2016b

¿Cómo se explica esta contradicción? De acuerdo con Waisbord (2014), algunas de las reformas emprendidas no necesariamente fueron por pluralizar y democratizar los sistemas de medios, sino en una utilización de lo mediático como un elemento central de una política basada en un liderazgo presidencial sin contrapesos. Desde la perspectiva de Vaidya y Gupta (2016), la comprensión de los medios como parte de un determinado proyecto político en nada ayuda a un ambiente propicio para la accountability de los medios.

Si bien no se pueden cuestionar todas las medidas llevadas a cabo por estos gobiernos de falsas intenciones, sobre todo en sistemas mediáticos dominados por lógicas comerciales y en donde lo público tiene muy baja incidencia, estas administraciones reformistas han levantado suspicacias respecto a sus propósitos de pluralizar los sistemas de medios (Gómez y Ramos-Martín, 2014, Waisbord, 2014) y en definitiva, aunque nunca propuesto como parte de sus objetivos, crear un ambiente propicio para una adecuada función de vigilancia mediática.

Pese a que todas estas reformas han tenido sus propias características de acuerdo con las realidades de los países en que se han realizado, cabe destacar, que Uruguay tras sus intentos de pluralización del sistema en 2014, sigue estando como una de las 
naciones con mayor libertad de prensa de la región (Freedom House, 2016a; Reporteros sin Fronteras, 2016a).

\section{Conclusiones}

Desde el retorno de la democracia a América Latina y dadas ciertas condiciones de libertad e independencia a los medios, hubo importantes casos, aunque a cuentagotas, en que el periodismo cumplió una labor importante para obligar a rendir cuentas al poder político ante la ciudadanía. Esto teniendo en consideración que siempre resulta complejo establecer las verdaderas intenciones políticas y económicas que motivaron estas denuncias, y que sus consecuencias siempre han resultado impredecibles.

Sin embargo, la existencia de estos casos de watchdog, más que plantear la existencia de una accountability mediática en la región, cuestiona todas las oportunidades en que los medios desde la Tercera Ola no han cumplido esta función, siendo incluso actores cuestionables en su actuar para con la democracia.

De esta manera, la competencia de más medios, más que la concentración en unos pocos, parece ser el ambiente más propicio para esta función mediática. Esto tanto, porque una mayor cantidad de puntos de vista promueve más la verdad y menos la manipulación, y hace más difícil la captura de la información por parte del poder político.

Es necesario considerar que las reformas a los sistemas de medios de comunicación en la región no solo deben ser desde la perspectiva de lograr un mayor pluralismo de la información, sino también para crear condiciones para que los medios puedan cumplir una de sus funciones más importantes y deseables en las democracias que es la de hacer rendir cuentas al poder político.

\section{Referencias bibliográficas}

Alcántara, M. (2012). Partidos políticos en América Latina: hacia una profesionalización de calidad. Convergencia, 19 (58), 53-70.

Alcántara, M. (2006). Los políticos y la política tras un cuarto de siglo de democracia. En Alcántara, M. (Ed.). Políticos y política en América Latina. Madrid: Siglo XXI.

Allen, D. y Blank Hindman, E. (2014). The media and democracy: Using Democratic Theory in Journalism Ethic. En Wyatt, W. (Ed.), The Ethics of Journalism: Individual, Institutional and Cultural Influences. Londres: University Oxford.

Amado Suarez, A. (2014). Calidad periodística y fuentes presidenciales: el periodismo argentino frente a la comunicación de gobierno. Estudios sobre el Mensaje Periodístico, 63, Vol. 21, 63-84.

Auat, L. (2003). La racionalidad política. Principios y mediaciones. Revista de filosofía de Santa Fe, 11, 45-61.

Badillo, Á., Mastrini, G., y Marenghi, P. (2015). Teoría crítica, izquierda y políticas públicas de comunicación: el caso de América Latina y los gobiernos progresistas. Comunicación y sociedad, 24, 95-126.

Becerra, M. (2014). Medios de comunicación: América Latina a contramano. Nueva Sociedad, 249, 61-74. 
Becerra, M. (2011). La disputa y sus matices (Prólogo). En De Moraes, D. (Ed.), La cruzada de los medios en América Latina. Gobiernos progresistas y políticas de comunicación. Buenos Aires: Paidós.

Becerra, M. y Mastrini, G. (2006). Periodistas y Magnates. Estructura y concentración de las industrias culturales en América Latina. Buenos Aires: Prometeo Libros.

Beltrán, R. (2008). Comunicación para la democracia en Iberoamérica. Paper presentado en El espacio Iberoamericano de comunicación en la era digital, Facultad de Comunicación de la Universidad de Sevilla.

Bertot, J., Jaeger, P. y Grimes, J. (2012). Promoting transparency and accountability through ICTs, social media, and collaborative e-government. Transforming Government: People, Process and Policy, 6, 78-91.

Besley, T. y Prat, A. (2006). Handcuffs for the grabbing hand? media capture and government accountability. American economic review, 96, 720-736.

Boas, T. (2012). Mass Media and Politics in Latin America. En Dominguez, J. y Shifter, M. (Eds.), Constructing Democratic Governance in Latin America. Baltimore: Johns Hopkins University Press.

Cañizales, A. y Matos-Smith, M. (2015). El caso de Globovisión y la implantación del modelo mixtoautoritario en el sistema de medios. Revista Iberoamericana, 15, 59, 127-140.

Cárdenas, F. y González, J. (2006). Los Watergates Latinos. Colombia: Ediciones B.

CEPAL (2016). Estado de la banda ancha en América Latina y el Caribe 2016. Comisión Económica para América Latina y el Caribe (CEPAL).

Curran, J. (2002). Media and Power. Estados Unidos: Routledge.

Christians, C., Glasser, T., McQuail, D., Nordenstreng, K., y White, R. (2009). Normative Theories of the Media. Journalism in democratic societies. Estados Unidos: University of Illinois.

Del Tronco, J. (2013). Desconfianza y accountability ¿Las causas del populismo en América Latina? Latin American Research Review, 48, 55-78.

EGM (2016). Estudio general de medios. Asociación Colombiana de Investigación de Medios: Colombia.

Fernández, B. (2014). Avisaje estatal: la tentación gubernamental de manipular los medios. El Mostrador, 4 de abril de 2014.

Foucault, M. (1992). El orden del discurso. Buenos Aires: Tusquet.

Foucault, M. (1979). Microfísica del poder. España: La Piqueta.

Fox, E. y Waisbord, S. (2002). "Introduction". En Fox, E. y Waisbord, S. (Eds.), Latin politic, global media. Estados Unidos: University of Texas Press.

Fox, J. (2015). Social Accountability: What Does the Evidence Really Say? World Development, 72, 346-361.

Freedom House (2016a). Freedom of the press 2016. Washington, DC: Freedom House.

Freedom House (2016b). Freedom of the press. Scores and Status Data 1980-2016. Washington, DC: Freedom House.

Freidenberg, F. (2007). La tentación populista. Una vía al poder en América Latina. Madrid: Síntesis.

Galarza, R. (2014). Clase política y medios: explorando el fenómeno de la publicidad política encubierta en México. Revista Mexicana de Ciencias Políticas y Sociales, 59, 51-76. 
Gentzkow, M y Shapiro, J. (2008). Competition and Truth in the Market for News. Journal of Economic Perspectives, 22, 133-154.

Gómez, A. y Ramos-Martín, J. (2014). Legislación y medios comunitarios. Análisis comparativo de Bolivia y Venezuela. Palabra Clave, 17,2, 484-516.

Goldstein, A. (2015). Los condicionamientos de la prensa en la crisis política del segundo mandato de Dilma Rousseff. Revista Política Latinoamericana, 1, 36-44.

Guerrero, M. (2014). The 'capture liberal' model of media system in Latin America. En Guerrero, M. y Márquez-Ramírez, M. (Eds.), Media Systems and Communication Policies in Latin America. Estados Unidos: Springer.

Hampton, M. (2010). "The Fourth Estate Ideal in Journalism History". En Stuart, A. (Ed.), The Routledge Companion to News and Journalism. Reino Unido: The Routledge Companion to News and Journalism.

Hallin, D. y Mancini, P. (2004). Comparando sistemas de medios de comunicación: Tres modelos de medios de comunicación y política. Reino Unido: Cambridge University Press.

Huges, S. (2006). Newsrooms in conflict: journalism and the democratization of Mexico. Pittsburgh: University of Pittsburgh Press.

Huntington, S. (1991). Democracy's Third Wave. The Journal of Democracy, 2, 12-34.

Instituto Verificador de Circulaciones (2016). Boletín X Press. Septiembre 2016. Instituto Verificador de Circulaciones: Argentina.

Kitzberger, P. (2016). Media Wars and the New Left: Governability and Media Democratisation in Argentina and Brazil. Journal of Latin America Studies, 48, 447-476.

Lasswell, H. (1948). The structure and function of communication in society. En Bryson, L. (Ed.), The Communication of Ideas. Nueva York: The Institute for Religious and Social Studies.

Latinobarómetro (2015). Informe 1995-2015. Santiago de Chile: Latinobarómetro.

Lippmann, W. (1922). Public Opinion. Estados Unidos: Brace.

Loreti, D. y Lozano, L. (2014). El derecho a comunicar. Los conflictos en torno a la libertad de expresión en las sociedades contemporáneas. Argentina: Siglo XXI.

Mainwaring, S. y Pérez-Liñán, A. (2014). Democracies and Dictatorships in Latin America: Emergence, Survival, and Fall. Estados Unidos: Cambridge University Press.

Mainwaring, S. (2003). Introducction: Democratic Accountability in América Latina. En Mainwaring, S. y Welna, C. (Eds.), Democratic Accountabilty in América Latina. Nueva York: Oxford University Express.

McQuail, D. (2013). Journalism an society. Estados Unidos: Sage.

Media Research Center (2016). Comportamiento de audiencia en TV abierta, 20 de octubre de 2016. Media Research Center: México.

Noam, E. y Mutter, P. (2016). Brazil-Data Sumaries. En Noam, E. (Ed.), Who Owns the World's Media? Media Concentration and Ownership Around the World. Oxford University Press: Nueva York.

O'Donnell, G. (2004). Notas sobre la democracia en América Latina. Programa de Naciones Unidas para el Desarrollo.

O'Donnell, G. (1996). Otra institucionalización. Política y gobierno, III, 219-244. 
O'Donnell, G. (1994). Delegative Democracy? Journal of Democracy, 5, 55-69.

Pasquino, G. (2015). "Los retos de la democracia en América Latina". Temas y debates, 29, 105-112.

Peruzzotti, E. (2006). Media scandals and social Accountability. Assessing the role of the senate scandal in Argentina. En Peruzzotti, E. y Smulovitz, C. (Eds.), Enforcing the rule of law: social Accountability in the new Latin American democracies. Estados Unidos: University of Pittsburgh Press.

Peruzzotti, E. y Smulovitz, C. (2006). Social Accountability: An introduction". En Peruzzotti, E. y Smulovitz, C. (Eds.), Enforcing the rule of law: social Accountability in the new Latin American democracies. Estados Unidos: University of Pittsburgh Press.

Peruzzotti, E. y Smulovitz, C. (2002). Civil society, the media and internet as tools for creating accountability to poor and disadvantaged groups. Programa de Naciones Unidas para el Desarrollo.

Prat, A. y Stromberg, D. (2013). The political economy of mass media. En Acemogli, D. Arellano, M. y Dekel E., Advances in Economics and Econometrics, Volume 2, Estados Unidos: Cambridge.

Reporteros sin Fronteras (2016a). Clasificación mundial 2016 de la libertad de prensa. La paranoia de los dirigentes frente a los periodistas. Reporteros sin Fronteras.

Reporteros sin fronteras (2016b). Medios de comunicación. Los oligarcas se van de compras. Reporteros sin Fronteras.

Salwen, M. y Garrison, B. (2014). Latin American Journalism, Estados Unidos: Routledge.

Santander, P. (2014). Nuevas leyes de medios en Sudamérica: enfrentando políticamente la concentración mediática. Convergencia, 22, 13-37.

Santoro, D. (2008). Técnicas de investigación. Métodos desarrollados en diarios y revista de América Latina. México: Fondo de Cultura Económica

Távara J. (2016). Economía política de la concentración de los medios. En ¿̇Puede acapararse la libertad? Libertad de expresión y concentración de medios en el Perú. Fundación para el Debido Proceso - Facultad de Derecho PUCP, Lima.

Television.com.ar (2016). Informe de rating diciembre 2016. Televisión.com.ar: Argentina.

Thompson, J. (2001). El escándalo político: poder y visibilidad en la era de los medios de comunicación. Barcelona: Paidós.

Tocqueville, A. (1835-1840). De la démocratique en América. México: Fondo de Cultura Económica.

Transparency International (2016). Corruption Perceptions Index 2015. Berlín, Alemania: Transparency International.

Trejo, R. (2010). Poderes salvajes. Mediocracia sin contrapesos. Cal y arena: México.

UNESCO (2014). Tendencias mundiales en libertad de expresión y desarrollo de los medios: Situación regional en América Latina y el Caribe. Uruguay: UNESCO.

Vaidya, S. y Gupta, R. (2016). Corruption Via Media Capture: The Effect of Competition. Southern Economic Journal, 82, 1327-1348.

Valida (2016). Boletines de circulación y lectura. Primer semestre 2016. Valida, Santiago de Chile.

Van Dijk, T. (2016). "Como el globo manipuló la distitución de Dilma Rousseff". Legcom. Lenguaje, sociedad y comunicación, 12,1 . 
Waisbord, S. (2014). Vox populista. Medios, periodismo, democracia. Barcelona: Gedisa.

Waisbord, S. (2012). Political Communication in Latin America. En Semetko, H. y Scammell, M. (Eds.), The SAGE Handbook of Political Communication. Estados Unidos: SAGE.

Waisbord, S. (2000). Watchdog journalism in South America. News, Accountability, and Democracy. Nueva York: Columbia University Press.

Waisbord, S. (1996). Investigative journalism and political accountability in South American democracies. Critical Studies in Mass Communication, 13, 343-363.

Weber, M. (1922). Wirtschaft und Gesellschaft. Grundriss der Vmtehendn Soziologie. Alemania: I.C.B. Mohr.

Weyland, K. (2010). The performance of Leftist Government in Latin America: Conceptual and Theorical Issues. En Weyland, K. Madrid, R. y Hunter, W. (Eds.), Leftist Governments in Latin America: Successes and Shortcomings. Reino Unido: Cambridge University Press. 